\title{
Overexpression of peroxiredoxin 2 inhibits TGF-ß1-induced epithelial-mesenchymal transition and cell migration in colorectal cancer
}

\author{
JIHONG FENG ${ }^{1}$, ZHONGXUE FU ${ }^{1}$, JINBAO GUO ${ }^{1}$, WEIDONG LU ${ }^{1}$, KUNMING WEN ${ }^{2}$, \\ WANGSHENG CHEN $^{3}$, HAO WANG ${ }^{1}$, JILAI WEI $^{1}$ and SHOURU ZHANG ${ }^{1}$
}

\author{
${ }^{1}$ Department of Gastrointestinal Surgery, The First Affiliated Hospital, Chongqing Medical University, Chongqing 400016; \\ ${ }^{2}$ The Affiliated Hospital, Zunyi Medical College, Zunyi, Guizhou 563003; ${ }^{3}$ The Affiliated Hospital, \\ Luzhou Medical College, Luzhou, Sichuan 266003, P.R. China
}

Received February 12, 2013; Accepted May 28, 2014

DOI: $10.3892 / \mathrm{mmr} .2014 .2316$

\begin{abstract}
Although human peroxiredoxin 2(PRDX2) has been implicated in tumor progression (e.g., invasion and metastasis), little is known regarding its role in the epithelial-mesenchymal transition (EMT) process during tumorigenesis. The present study offers the first evidence, to the best of our knowledge, that the antioxidant enzyme PRDX2 has an important role in regulating the EMT process. It was demonstrated that overexpression of PRDX2 leads to changes in cell morphology in vitro and potent inhibition of the transforming growth factor (TGF)- $\beta 1$-induced EMT and cell migration of colorectal cancer (CRC) cells. Furthermore, PRDX2 regulates the expression of EMT markers, EMT-related transcription factors and metastasis-related factors in CRC cells. These results provide new insight into the role of PRDX2 in regulating EMT, cell migration and metastasis of CRC cells. It was concluded that the upregulation of PRDX2 may be correlated with EMT and contributes to the pathogenesis of CRC by inhibiting EMT, cell migration and metastasis. Taken together, these findings suggest that PRDX2 may be a key regulator of invasion and metastasis by inhibiting EMT of CRC cells, and also identifies a therapeutic strategy to effectively decrease the lethality of highly malignant types of CRC.
\end{abstract}

\section{Introduction}

Colorectal cancer (CRC), which mainly originates from the intestinal epithelium as premalignant lesions, termed adenomas, is one of the most commonly diagnosed cancers

Correspondence to: Professor Zhongxue Fu, Department of Gastrointestinal Surgery, The First Affiliated Hospital, Chongqing Medical University, No. 1 Medical College Road, Chongqing 400016, P.R. China

E-mail: fuzhongxue2014@163.com

Key words: peroxiredoxin 2, epithelial-mesenchymal transition, transforming growth factor- $\beta 1$, colorectal cancer in males and females worldwide (1). Despite prompt surgical removal followed by adjuvant therapy, which is often suitable in the early stages of the disease, the majority of patients still undergo therapeutic resistance and recurrence. Therefore, investigation of the genetic and molecular mechanisms that contribute to the progression and metastasis of CRC is urgently required.

Previous evidence suggests that cancer progression and metastasis are tightly associated with epithelial-mesenchymal transition (EMT). EMT is a developmental process that involves actin cytoskeleton reorganization and the loss of apical-basal polarity and cell-to-cell contact, resulting in the conversion of epithelial cells to mesenchymal cells $(2,3)$. Cells undergoing EMT are characterized by the loss of E-cadherin and other components of epithelial cell junctions (4), and the acquisition of mesenchymal markers, including $\mathrm{N}$-cadherin and Vimentin (5). A series of EMT-inducing transcription factors, including the zinc-finger proteins Snail and Slug, the bHLH factor Twist, the zinc-finger/homeodomain proteins ZEB1 and ZEB2, and the forkhead factor FoxC3, have been associated with tumor invasion and metastasis (6). Twist1, like other EMT-inducing transcription factors, including Snail, Slug and SIP1, binds DNA through similar E-box sequence motifs and represses E-cadherin and other epithelial cell adhesion molecules. Further studies on different hepatic cellular cancer (HCC) cell lines revealed that Twist1 is able to downregulate E-cadherin expression and promote matrix metalloproteinase (MMP) activation, specifically in MMP2 and MMP9 (7). Transforming growth factor (TGF)- $\beta 1$ is a multi-functional cytokine with diverse effects on cancer cells (8). TGF- $\beta 1$ is perhaps the most common inducer of EMT $(9,10)$. Although stimuli, including TGF $\beta$, FGF, EGF, IGF, HGF, PDGF, estrogens, Wnt, Shh, inflammatory cytokines or hypoxia, as well as oncogenes like Ras ${ }^{\mathrm{V} 12}$, ErbB2 or mutant p53, may induce EMT during cancer progression (3), the triggering and maintenance of EMT is mainly orchestrated by three major groups of transcription factors: the ZEB, Snail and Twist families (11).

Peroxiredoxins (PRDXs) constitute a family of antioxidant enzymes that control cytokine-induced peroxide levels, which mediate signal transduction in mammalian cells (12). As cancer 
cells are known to produce large amounts of reactive oxygen species (ROS), it is readily appreciated that overexpression of the antioxidant enzyme, peroxiredoxins, may be beneficial for cancer cell survival. In addition to the importance of eliminating oxidants, recent evidence has begun to implicate other specific roles of antioxidant enzymes in cellular functions, via protein-protein interaction or controlling the local redox environment (13). However, the functions of peroxiredoxins are not restricted to their antioxidant activities. A number of studies have revealed novel functions in self-defense against infection, tissue damages and tumors, through the regulation of inflammation (14).

PRDX2, which is a typical 2-Cys thioredoxin peroxidase and a cellular antioxidant that is widely distributed in tissues, has been demonstrated to be overexpressed in various types of cancer cells and tumor tissues. By virtue of its role in maintaining the redox state in vivo, PRDX2 is a prime candidate for regulating the $\mathrm{H}_{2} \mathrm{O}_{2}$ signaling that is initiated by cell surface receptors (15). Previously, it has been well demonstrated that PRDX2 may have a protective role against skin aging and cellular senescence (16). Further characterization has revealed that PRDX2 is present in the nucleus and enhances agent-induced activation of the JNK/c-Jun pathway involved in repair of damaged DNA (17). By contrast, PRDX2 was recently demonstrated to function as a negative regulator of PDGF signaling by suppressing the proliferation and migration of smooth muscle cells (SMCs) (18). Another study also suggested that PRDX2 acts as a growth suppressor to slow the induction of leukemia caused by the c-Myc oncogene (19). These studies indicate that PRDX2 may be applicable as not only a predictive biomarker, but also a potential therapeutic target.

However, to the best of our knowledge, evidence of a possible correlation between PRDX2 expression and the EMT process has not been published. The results from the present study indicated that PRDX2 negatively regulates this process through a pathway involving the transcription factors Twist1, Snail, ZEB1 and ZEB2. Furthermore, the results demonstrated that upregulation of PRDX2 may be correlated with EMT and contribute to the pathogenesis of CRC by inhibiting EMT, cell migration and metastasis.

\section{Materials and methods}

Cell culture. The colorectal cancer cell lines, SW480 and SW620, were respectively derived from tumor stage Dukes' type B and C human colorectal adenocarcinoma, which were both purchased from American Type Culture Collection (ATCC, Manassas, VA, USA) and maintained in Leibovitz's 15 (L-15) cell culture medium (Gibco, Carlsbad, CA, USA) supplemented with $10 \%$ fetal bovine serum (FBS; SH30084.03; Hyclone, Logan, UT, USA) at $37^{\circ} \mathrm{C}$ in a humidified atmosphere with $5 \%(\mathrm{v} / \mathrm{v}) \mathrm{CO}_{2}$.

Reagents and antibodies. Recombinant human TGF- $\beta 1$ (AF-100-21C) was purchased from PeproTech (Rocky Hill, NJ, USA). The anti-hPRDX2 antibody (EPR5154) was obtained from Abcam (Cambridge, MA, USA). Rabbit monoclonal anti-human antibodies to E-cadherin (CDH1), N-cadherin (CDH2), Vimentin (EPR3776), MMP2 (EPR1184) and MMP9 (EP1254) were purchased from Epitomics (Abcam, San
Francisco, CA, USA). Anti-human antibodies against Twist1 (H-81), Snail1 (H-130) and Slug (A-7) were obtained from Santa Cruz Biotechnology, Inc. (Santa Cruz, CA, USA). Mouse monoclonal anti-human GAPDH and $\beta$-actin antibodies were obtained from Tianjin Sungene Biotech, Co., Ltd. (Tianjin, China). The HRP-conjugated goat anti-mouse or anti-rabbit antibodies were purchased from Beyotime Institute of Biotechnology (Shanghai, China). DyLight ${ }^{\mathrm{TM}} 488$ AffiniPure goat anti-rabbit secondary antibody and 4,6-diamidino-2-phenylindole dihydrochloride (DAPI; blue fluorescence) were purchased from EarthOx (San Francisco, CA, USA). The SYBR Green Quantitative PCR kit was obtained from Takara (Dalian, China).

Cloning and transfection. The coding region of the human PRDX2 gene was cloned from a human cDNA library (GenBank Accession no. NM_005809), full-length PRDX2 cDNA was polymerase chain reaction (PCR)-amplified using the following oligonucleotide primers: PRDX2-F: 5'-GAGGATCCCCGGGTACCGGTCGCCA

C C A T G G C C T C C G G T A A C G C - 3' PR DX 2 - R : 5'-TCACCATGGTGGCGACCGGATTGTGTTTGGAGAAA

TATTCC-3'. Briefly, the resulting fragment was ligated into the pUbi-MCS-EGFP-IRES-Puromycin plasmid (Shanghai GeneChem, Shanghai, China) to produce the lentiviral vector pUbi-PRDX2-MCS-EGFP-IRES-Puromycin, which was named LV-PRDX2. As a control, a lentiviral vector that expresses EGFP alone was also generated and was named Vector or LV-CON. The final recombinant lentiviral construct also was confirmed by DNA sequencing analysis.

When the cells reached $\sim 70-80 \%$ confluence, they were transfected by Lipofectamine 2000 (Invitrogen Life Technologies, Carlsbad, CA, USA) in accordance to the manufacturer's instructions. At $72 \mathrm{~h}$ post-transfection, the cells were observed under a fluorescence microscope and the functional overexpression of PRDX2 was confirmed by western blot analysis.

Cell migration assay. Cell migration assays were performed using a two-chamber transwell device (Costar, Corning, USA) according to the manufacturer's instructions. After the cells were transfected by LV-PRDX2 or LV-CON for $96 \mathrm{~h}, 50,000$ cells that were suspended in L-15 medium containing $0.1 \%$ FBS were seeded onto the upper chamber of the device, and $500 \mu \mathrm{l}$ of L-15 medium containing $10 \%$ FBS was added to the lower chamber; the device was incubated at $37^{\circ} \mathrm{C}$ for $36 \mathrm{~h}$. The inner side of the upper chamber was then wiped with a wet cotton swab to remove the cells, while the outer side of the chamber was gently rinsed with phosphate-buffered saline (PBS) and treated with 4\% paraformaldehyde for $20 \mathrm{~min}$. The membrane was then rinsed with PBS again and stained with a $0.1 \%$ crystal violet staining solution for $30 \mathrm{~min}$. After drying, >3 fields (between 3 and 8 fields) of the membrane were photographed, and the cells that migrated through the membrane were counted in the lower wells of the chamber by Countess ${ }^{\circledR}$ Automated Cell Counter (Invitrogen Life Technologies). Each experiment was performed three times.

RNA isolation and quantitative polymerase chain reaction ( $q P C R)$. Total RNA was isolated using TRIzol reagent (Invitrogen Life Technologies). Total RNA concentration was determined spectrophotometrically at 260 and $280 \mathrm{~nm}$. 
cDNA was synthesized using the TaqMan miRNA Reverse Transcription kit (Qiagen, Valencia, CA, USA). The mRNA level was determined using the SYBR PrimeScript RT-PCR kit (Takara).

qPCR was performed using the QPCR SYBR Green Low ROX Mix (Thermo Fisher Scientific Inc., Rockford, IL, USA) according to the manufacturer's instructions. The mRNA expression levels of the target genes and GAPDH as a normalizing control were analyzed using the following primer sets: E-cadherin, forward: 5'-CTTCTCTC ACGCTGTGTCATC-3' and reverse: 5'-CTCCTGTGTTCC TGTTAATGGT-3'; N-cadherin, forward: 5'-CGAATGGATG AAAGACCCATCC-3' and reverse: 5'-GGAGCCACTGCC TTCATAGTCAA-3'; Vimentin, forward: 5'-AGGCAAAGC AGGAGTCCA-3' and reverse: 5'-TATCAACCAGAGGG AGTG-3'; Twist1, forward: 5'-GGCTCAGCTACGCCTTC TC-3' and reverse: 5'-TCCTTCTCTGGAAACAATGACA-3'; Snail, forward: 5'-GACCACTATGCCGCGCTCTT-3' and reverse: 5'-TCGCTGTAGTTAGGCTTCCGATT-3'; Slug, forward: 5'-TGGTTGCTTCAAGGACACAT-3' and reverse: 5'-GCAAATGCTCTGTTGCAGTG-3'; ZEB1, forward: 5'-TACAGAACCCAACTTGAACGTCACA-3' and reverse 5'-GATTACACCCAGACTGCGTCACA-3'; ZEB2, forward: 5'-CACAGCTCTTCCACCTCAAAGC-3' and reverse: 5'-TTTTGCGAGACAGACAGGAG-3' and GAPDH, forward: 5'-GCACCGTCAAGGCTGAGAAC-3' and reverse: 5'-TGGTGAAGACGCCAGTGGA-3'. Thermal cycling conditions comprised 1 step for $10 \mathrm{~min}$ at $95^{\circ} \mathrm{C}$ followed by 40 cycles for $15 \mathrm{sec}$ at $95^{\circ} \mathrm{C}$ and for $1 \mathrm{~min}$ at $60^{\circ} \mathrm{C}$. Each measurement was performed in duplicates and the PCR product quality was monitored through post-PCR melt-curve analysis. The relative values of gene expression were normalized to that of GAPDH and calculated using the $2^{-\Delta \Delta \mathrm{Ct}}$ method, where $\Delta \Delta \mathrm{Ct}=$ $\left(\Delta \mathrm{Ct} \text { target gene }-\Delta \mathrm{Ct}_{\mathrm{GAPDH}}\right)_{\text {sample }}-\left(\Delta \mathrm{Ct}_{\text {target gene }}-\Delta \mathrm{Ct}_{\mathrm{GAPDH}}\right)_{\text {control }}$. The fold change in relative expression was then determined by calculating $2^{-\Delta \Delta \mathrm{Ct}}$.

Western blot analysis. Total proteins were isolated from frozen tissues using lysis buffer (50 mM Tris pH 7.5, $150 \mathrm{mM} \mathrm{NaCl}$, $10 \mathrm{mM}$ EDTA, $1 \%$ NP-40, 0.1\% SDS, $1 \mathrm{mM}$ PMSF and $0.5 \%$ sodium deoxycholate), and quantified using the Bradford method. The cell lysates were separated on 8 or $12 \%$ SDS-PAGE gel and transferred onto a PVDF membrane at $90 \mathrm{~V}$ for $150 \mathrm{~min}$. The blots were incubated with anti-E-cadherin, anti-N-cadherin, anti-Vimentin, anti-Twist1, anti-Snail, anti-Slug, anti-MMP2 and anti-MMP9 antibodies and developed using the enhanced chemiluminescence (ECL) detection system (Bio-Rad, Hercules, CA, USA). The same blot was stripped and reprobed with the anti- $\beta$-actin antibody for use as an internal control.

Statistical analysis. Data are presented as the mean \pm standard deviation of three independent experiments, and the SPSS version 18 software package was used for statistical analysis (SPSS, Inc., Chicago, IL, USA). P $<0.05$ was considered to indicate a statistically significant difference.

\section{Results}

Forced PRDX2 expression blocks TGF- $\beta 1$-induced EMT and cell migration. To investigate whether PRDX2 is involved in
TGF- $\beta 1$-induced EMT, its expression was transiently forced in CRC cells using lentivirus vectors. LV-PRDX2 or LV-CON were transfected into colorectal cancer SW480 cells, then the morphological changes were observed with phase-contrast microscopy and the expression of PRDX2 was detected by western blotting (Fig. 1A and C). Compared with the untreated cells (Con) and lentiviral vector control (Vector), the SW480 cells transfected by LV-PRDX2 exhibited a change of cell accumulation. As observed, the SW480 cells treated with TGF- $\beta 1$ for $72 \mathrm{~h}$ lead to a morphological change from an epithelial phenotype to an elongated, spindle-like mesenchymal phenotype. These findings demonstrated that TGF- $\beta 1$ may induce EMT-like morphological changes in SW480 cells. Compared with the control group, the spindle-like morphological changes were not observed upon TGF- $\beta 1$ addition in LV-PRDX2 transfected cells (Fig. 1B). The result demonstrated that PRDX2 overexpression eliminated the EMT-like morphological changes of the SW480 cells.

To determine the role of PRDX2 during the EMT-like process, cell migration assays were performed (Fig. 1D). Further analysis demonstrated that forced PRDX2 expression markedly reduced the TGF- $\beta 1$-increased cell migration in the two cell lines compared with the control group $(\mathrm{P}<0.01$; Fig. 1E). Collectively, these results demonstrated that forced PRDX2 may inhibit TGF- $\beta 1$-induced EMT-like phenotype and cell migration in colorectal cancer cells.

PRDX2 regulates the expression of EMT markers and EMT-related transcription factors in colorectal cancer cells at the $m R N A$ level. To determine the function of PRDX2 during the EMT-like process in vitro, qPCR analysis for EMT markers and EMT-related transcription factors was performed. As demonstrated in Fig. 2A, the mRNA level of E-cadherin was markedly higher compared with the control group in the SW480 and SW620 cells. However, the mRNA level of the mesenchymal markers, $\mathrm{N}$-cadherin and Vimentin, were significantly reduced in comparison with those in the control cells. Compared with the control group, overexpression of PRDX2 significantly increased the mRNA level of the EMT-related transcription factors Twist1, Snail, Slug, ZEB1 and ZEB2 in CRC cells stimulated with TGF- $\beta 1$ for $72 \mathrm{~h}$ (Fig. 2B). These results revealed that PRDX2 regulates EMT markers and EMT-related transcription factors in CRC cells at the mRNA level.

Overexpression of PRDX2 modulates the expression of TGF- $\beta 1$-induced EMT markers and EMT-related transcription factors and metastasis-related factors. To investigate the molecular mechanism of how the overexpression of PRDX2 modulates the expression of TGF- $\beta 1$-induced EMT, the expression of EMT marker, EMT master transcription factors and metastasis-related factors was examined using western blot analysis. As predicted, the overexpression of PRDX2 resulted in the upregulation of the E-cadherin and downregulation of the $\mathrm{N}$-cadherin and Vimentin. Furthermore, its overexpression inhibited the expression of the transcription factors Twist1, Snail, Slug and metastasis-related factors MMP2 and MMP9 in CVC cells stimulated with TGF- $\beta 1$ (Fig. 3). Next, the regulatory effects of PRDX2 on EMT markers and the transcription factors Twist 1 were further confirmed using 
A

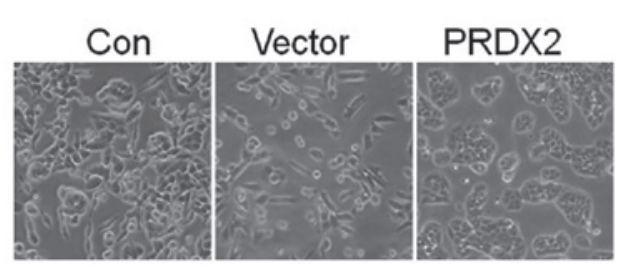

B Con

TGF- $\beta 1$ +
Vector -
PRDX2 -

Vector

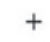

$+$
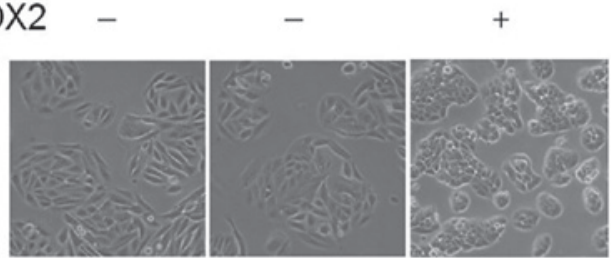

D

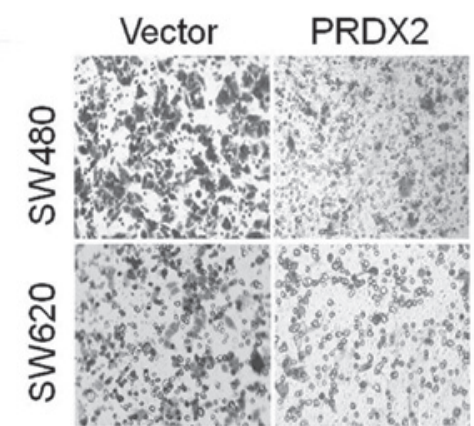

PRDX2 $\beta$-actin
$\mathbf{E}$

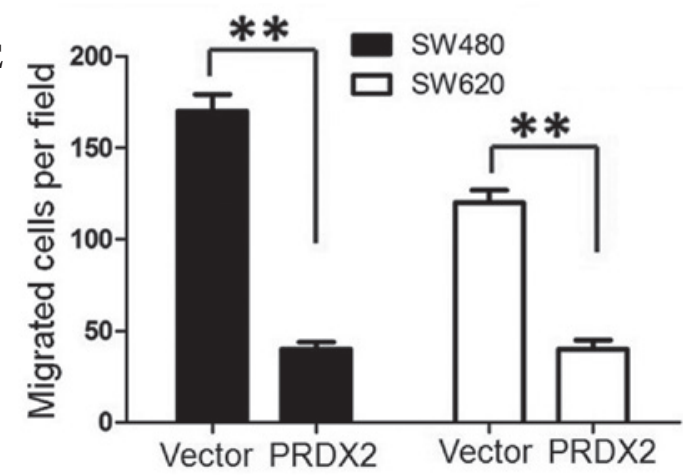

Figure 1. Overexpression of PRDX2 represses TGF- $\beta 1$-induced EMT and cellular migration in CRC cells. (A) Morphological changes of SW480 cells cultured under different conditions were analyzed by an inverted microscope. Con: negative control; Vector: lentiviral vector control; PRDX2: LV-PRDX2. (B) SW480 cells were treated with TGF- $\beta 1(5 \mathrm{ng} / \mathrm{ml})$ for $72 \mathrm{~h}$, the cell morphological changes associated with EMT are demonstrated in the phase contrast image. (C) SW480 cells were transfected by LV-PRDX2 or LV-CON (vector) for $96 \mathrm{~h}$, the expression of PRDX2 was analyzed by western blotting. $\beta$-actin served as the loading control. (D) Migration assay of the SW480 and SW620 cells. The cells transfected with LV-PRDX2 or LV-CON were stimulated with TGF- $\beta 1$ (5 ng/ $\mathrm{ml}$ ) for $48 \mathrm{~h}$, and the morphological changes were observed with a phase-contrast microscopy (magnification, x100). (E) The number of cells that migrated to the lower surface of the membrane per field was counted and quantified (magnification, $\mathrm{x} 200$ ). ${ }^{* *} \mathrm{P}<0.01$ vs. Vector. PRDX2, peroxiredoxin 2 ; TGF- $\beta 1$, transforming growth factor- $\beta 1$; EMT, epithelial-mesenchymal transition; CRC, colorectal cancer.

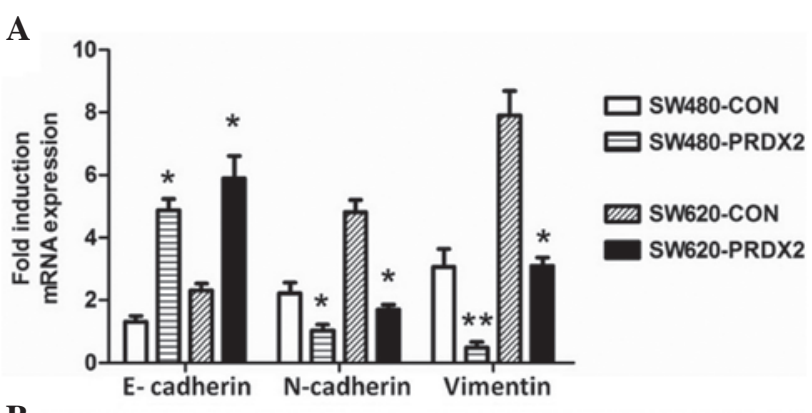

B

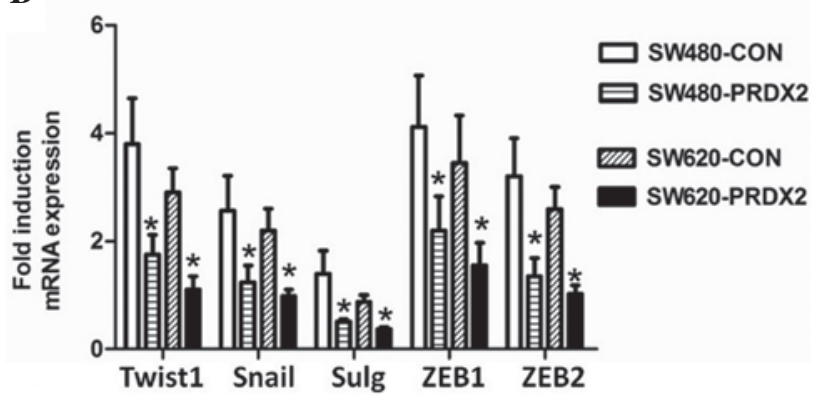

Figure 2. Upregulation of PRDX2 regulates the expression of EMT markers and EMT-related transcription factors in SW480 and SW620 cells stimulated with TGF- $\beta 1$ for $72 \mathrm{~h}$. (A) The expression levels of E-cadherin, N-cadherin and Vimentin mRNA were analyzed by qPCR. (B) The expression levels of Snail, Slug, ZEB1 and ZEB2 mRNA were analyzed by qPCR. The results are shown as the fold changes compared with those of LV-Control-transfected cells. "P $<0.05$, compared with the control. PRDX2, peroxiredoxin 2; TGF- $\beta 1$, transforming growth factor- $\beta 1$; EMT, epithelial-mesenchymal transition. immunofluorescence staining. The results suggested that PRDX2 may have an important role in the EMT-like process by upregulating E-cadherin and downregulating Vimentin and Twistl (Fig. 4). Together, these data further indicated that overexpression of PRDX2 modulates TGF- $\beta 1$-induced EMT in CRC cells.

\section{Discussion}

In the past decade, numerous studies have reported that the EMT process is a morphological event that is crucial to tumor migration and invasion in physiological and pathological states $(20,21)$. TGF- $\beta 1$ has an important role in tumor progression by inducting EMT $(4,22)$. Although it is well established that PRDX2 is involved in cancer progression, little is known regarding the function of PRDX2 in the EMT-like process of tumor cells.

In the present study, it was identified that overexpression of PRDX2 inhibits CRC cell invasion and TGF- $\beta 1$-induced EMT. Therefore, the upregulation of PRDX2 may cause MET in CRC cells, which is the reverse process of EMT, and associated with cell migration and metastasis. Although the cell morphological changes associated with EMT in PRDX2 knockdown cells were not studied, the present data suggested that forced PRDX2 expression modulates the expression of TGF- $\beta 1$-induced EMT markers and its related 


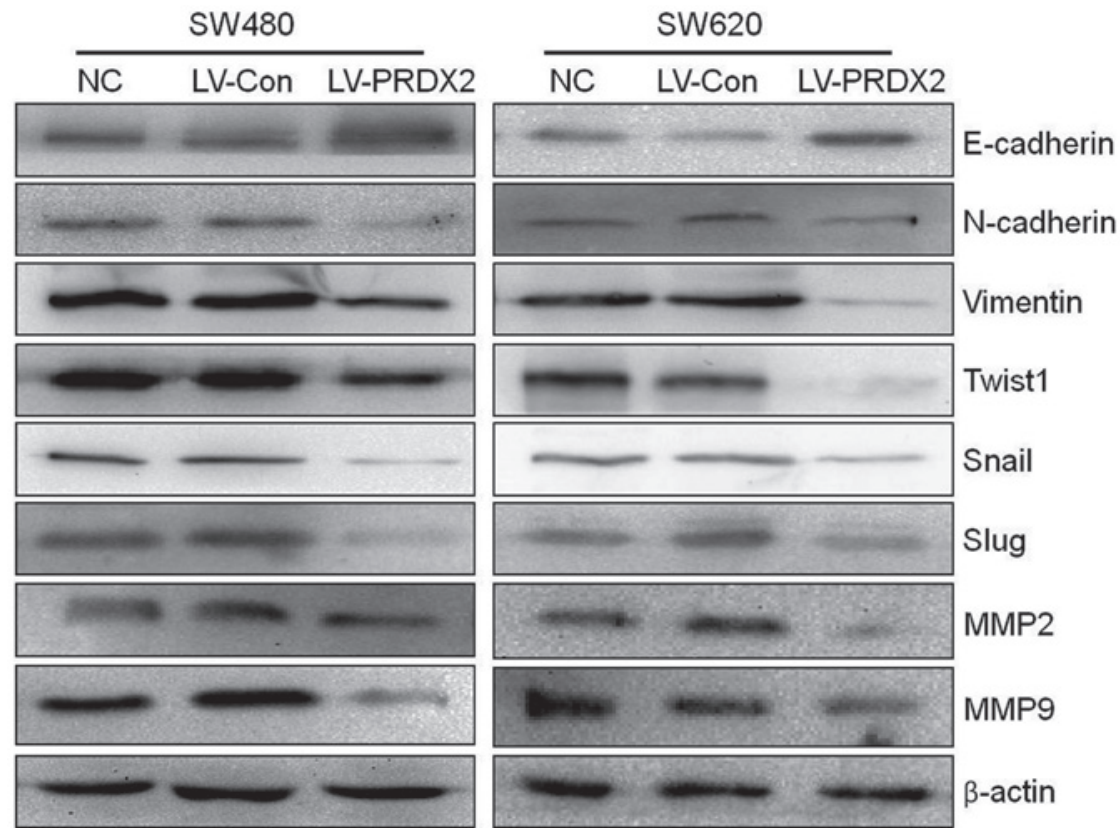

Figure 3. Expression of E-cadherin, N-cadherin, Vimentin, Twist1, Snail, Slug, MMP2 and MMP9 were analyzed by western blotting, and $\beta$-actin was used as a loading control. NC, negative control; LV-Con, lentiviral vector control; PRDX2, peroxiredoxin 2; MMP, matrix metalloproteinase.

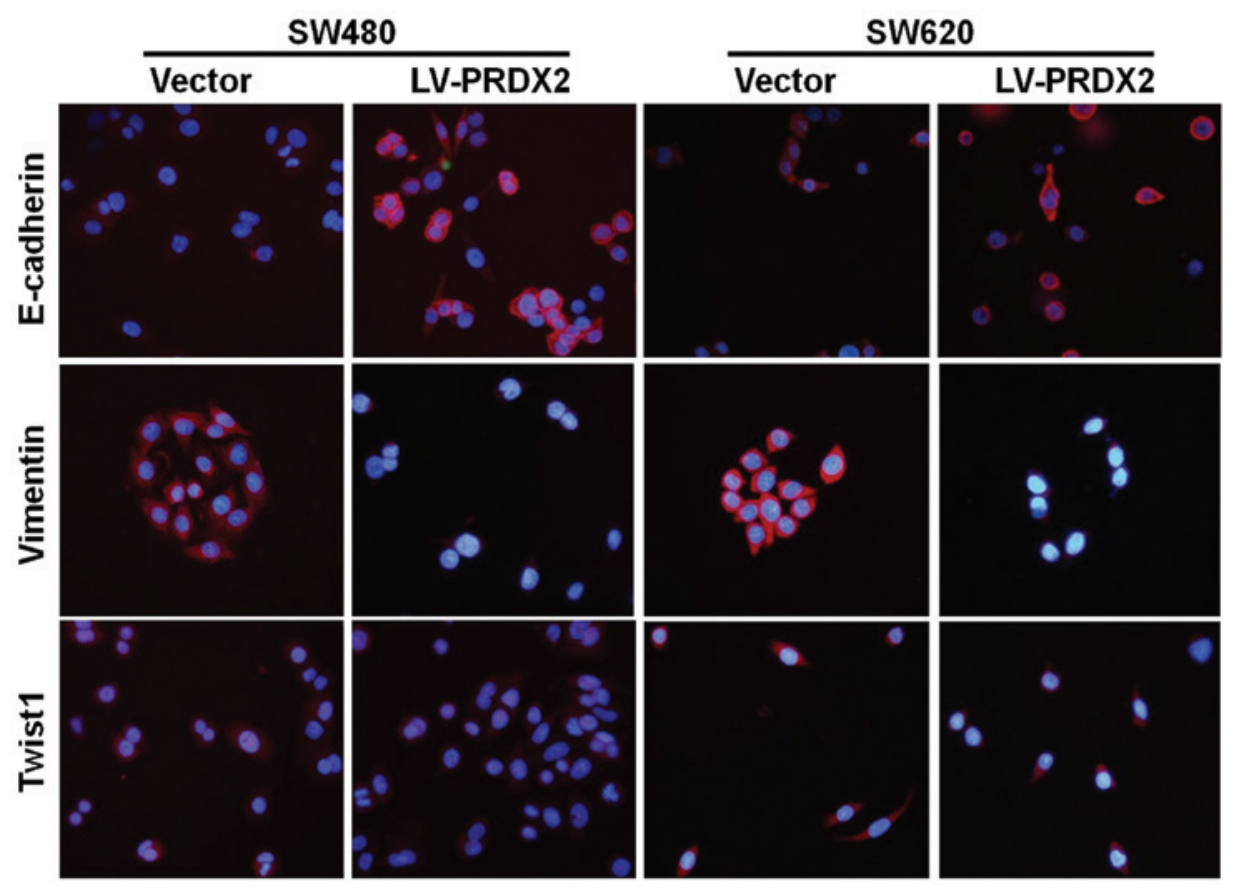

Figure 4. The expression of E-cadherin, Vimentin and Twist1 in LV-PRDX2-transfected or LV-Control-transfected cells were detected using immunofluorescence staining and phase-contrast microscopy. The cells were stained for E-cadherin, Vimentin or Twist1 (Red) and counter-stained with DAPI (blue; magnification, x200). PRDX2, peroxiredoxin 2 .

transcription factors, which indicates a potential role of PRDX2 in TGF- $\beta 1$-mediated biological functions. In addition, PRDX2 negatively regulated the metastasis-related factors, MMP2 and MMP9, suggesting that PRDX2 may be a new regulator of metastasis in $\mathrm{CRC}$ patients.

Previously, the expression of peroxiredoxins in mammalian cells, particularly peroxiredoxin1 (PRDX1) and PRDX2, has been considered to be indicative of signal peroxidases receiving, transducing and transmitting peroxide signals (23-25). However, a similar response of the ASK1-p38 pathway in knocking down PRDX1 and overexpressing PRDX2 suggested that the two cytosolic peroxiredoxins have distinct roles in the cellular peroxide response (23). Previously, one study reported that PRDX1 overexpression may enhance the TGF- $\beta 1$-induced EMT and cell migration, whereas knockdown of PRDX1 significantly inhibited the TGF- $\beta 1$-induced EMT and cell migration in lung cancer cells (26). These findings proved that PRDX2 
and PRDX1 may have opposing roles in the regulation of the TGF- $\beta 1$-induced EMT and cell migration in cancer cells. Further investigations are required to determine the molecular link between the two 2-Cys peroxiredoxins and EMT-related transcription factors.

It has recently been demonstrated that the progression from normal intestinal mucosa to adenoma (adenomatous mucosa) and finally to adenocarcinoma in CRC is closely correlated with the EMT process and changes in the expression of a series of genes, including E-cadherin, Vimentin and $\beta$-catenin (27). Another study revealed that PRDX2 may be a novel invasion and metastasis suppressor due to its ability to increase the formation of E-cadherin/ $\beta$-catenin complexes in melanoma (28). Our previous studies demonstrated that overexpression of PRDX2 promoted cell proliferation and prevented oxidation-induced apoptosis (29), whereas silencing of PRDX2 expression led to increased apoptosis and decreased proliferation by the $\mathrm{Wnt} / \beta$-catenin pathway in CRC cells (30). Furthermore, upregulation of PRDX2 resulted in the elimination of intracellular $\mathrm{H}_{2} \mathrm{O}_{2}$ (31), which negatively modulated the Wnt signal pathway by downregulating $\beta$-catenin (32). Key targets of the pathways that induce EMT include the adherens junction components E-cadherin and $\beta$-catenin. Therefore, PRDX 2 may be involved in cell adhesion by stabilizing the E-cadherin/ $\beta$-catenin complexes, and overexpression of PRDX2 may repress EMT and cell migration by activating the $\mathrm{Wnt} / \beta$-catenin pathways. This hypothesis is consistent with the behavior of PRDX2 in modulating the EMT signal pathway. In vivo and in vitro model systems have allowed the characterization of various pathways leading to EMT and EMT-like phenotypes. Downregulation of E-cadherin is a critical initial step in EMT, not only because of the disruption of adherens junctions but also because loss of E-cadherin reinforces the EMT process by inducing the expression of Twist1 and ZEB1 in a feed-forward loop (33). As EMT-activating transcription factors, particularly Snail, Slug, Twist, SIP1/ZEB are able to negatively regulate the expression of E-cadherin (34). Although the transcriptional regulatory networks that orchestrate the EMT process remain unclear, it is possible that PRDX2 may regulate the signaling pathways that induce E-cadherin regulators and modulate the EMT-related transcriptional factors. Extensive functional studies are therefore required to select candidate proteins for further validation.

In conclusion, although the mechanism of PRDX2 that is involved in the EMT-like process has not been fully illustrated, the data implicated PRDX2 in transcriptional regulation of the TGF- $\beta 1$-induced EMT and cell migration. The present findings demonstrated that PRDX2 may have a crucial role by impeding EMT in CRC cells, and implies that it represses cell migration and metastasis of CRC cells by controlling the expression of genes that are crucial for the TGF $\beta 1$-induced EMT. In addition, when the specific agents involved in the process of metastasis by inducing EMT are further identified, therapeutic strategies may be developed that target silencing of oncogenes or upregulating the EMT-mediated gene. Designing therapeutics targeting PRDX2 may offer a novel strategy for developing treatments for improving the prognosis for highly malignant types of CRC.

\section{Acknowledgements}

This study was supported by a grant from the Natural Science Foundation of China (grant no. 81172295). The authors are grateful to the editors of AJE for kindly revising our paper.

\section{References}

1. Siegel R, Naishadham D and Jemal A: Cancer statistics, 2012. CA Cancer J Clin 62: 10-29, 2012.

2. Yang $J$ and Weinberg RA: Epithelial-mesenchymal transition: at the crossroads of development and tumor metastasis. Developmental Cell 14: 818-829, 2008.

3. Thiery JP, Acloque H, Huang RY and Nieto MA: Epithelial-mesenchymal transitions in development and disease. Cell 139: 871-890, 2009.

4. Massagué J: TGFbeta in Cancer. Cell 134: 215-230, 2008.

5. Stefan Grünert MJHB: Diverse cellular and molecular mechanisms contribute to epithelial plasticity and metastasis. Nat Rev Mol Cell Biol 4: 657-665, 2003.

6. Voulgari A and Pintzas A: Epithelial-mesenchymal transition in cancer metastasis: mechanisms, markers and strategies to overcome drug resistance in the clinic. Biochim Biophys Acta 1796: 75-90, 2009.

7. Zhao XL, Sun T, Che N, et al: Promotion of hepatocellular carcinoma metastasis through matrix metalloproteinase activation by epithelial-mesenchymal transition regulator Twist1. J Cell Mol Med 15: 691-700, 2011.

8. Cho KH, Jeong KJ, Shin SC, Kang J, Park CG and Lee HY: STAT3 mediates TGF-beta1-induced TWIST1 expression and prostate cancer invasion. Cancer Lett 336: 167-173, 2013.

9. Kalluri R WR: The basics of epithelial-mesenchymal transition. J Clin Invest 119: 1420-1428, 2009.

10. Akhurst RJ and Derynck R: TGF-beta signaling in cancer - a double-edged sword. Trends Cell Biol 11: S44-S51, 2001.

11. Sánchez-Tilló E, Liu Y, de Barrios O, et al: EMT-activating transcription factors in cancer: beyond EMT and tumor invasiveness. Cellular and molecular life sciences. Cell Mol Life Sci 69: 3429-3456, 2012.

12. Wood ZA SE, Schröder E, Robin Harris J and Poole LB: Structure, mechanism and regulation of peroxiredoxins. Trends Biochem Sci 28: 32-40, 2003.

13. Kang DH, Lee DJ, Lee KW, et al: Peroxiredoxin II is an essential antioxidant enzyme that prevents the oxidative inactivation of VEGF receptor-2 in vascular endothelial cells. Mol Cell 44: 545-558, 2011.

14. Ishii T, Warabi E and Yanagawa T: Novel roles of peroxiredoxins in inflammation, cancer and innate immunity. J Clin Biochem Nutr 50: 91-105, 2012.

15. Rhee SG, Chae HZ and Kim K: Peroxiredoxins: a historical overview and speculative preview of novel mechanisms and emerging concepts in cell signaling. Free Radic Biol Med 38: 1543-1552, 2005.

16. Han YH, Kim HS, Kim JM, Kim SK, Yu DY and Moon EY: Inhibitory role of peroxiredoxin II (Prx II) on cellular senescence. FEBS Letters 579: 4897-4902, 2005.

17. Lee KW, Lee DJ, Lee JY, Kang DH, Kwon J and Kang SW: Peroxiredoxin II restrains DNA damage-induced death in cancer cells by positively regulating JNK-dependent DNA repair. J Biol Chem 286: 8394-8404, 2011.

18. Choi MH, Lee IK, Kim GW, et al: Regulation of PDGF signalling and vascular remodelling by peroxiredoxin II. Nature 435: 347-353, 2005.

19. Agrawal-Singh S, Isken F, Agelopoulos K, et al: Genome-wide analysis of histone $\mathrm{H} 3$ acetylation patterns in AML identifies PRDX2 as an epigenetically silenced tumor suppressor gene. Blood 119: 2346-2357, 2012.

20. Tiwari N, Gheldof A, Tatari M and Christofori G: EMT as the ultimate survival mechanism of cancer cells. Semin Cancer Biol 22: 194-207, 2012.

21. Wang Y and Shang Y: Epigenetic control of epithelial-to-mesenchymal transition and cancer metastasis. Exp Cell Res 319: 160-169, 2013.

22. Ko H, So Y, Jeon H, et al: TGF-beta1-induced epithelial-mesenchymal transition and acetylation of Smad2 and Smad3 are negatively regulated by EGCG in human A549 lung cancer cells. Cancer Lett 335: 205-213, 2013. 
23. Jarvis RM, Hughes SM and Ledgerwood EC: Peroxiredoxin functions as a signal peroxidase to receive, transduce, and transmit peroxide signals in mammalian cells. Free Radic Biol Med 53: 1522-1530, 2012.

24. Manandhar G, Miranda-Vizuete A, Pedrajas JR, et al: Peroxiredoxin 2 and peroxidase enzymatic activity of mammalian spermatozoa. Biol Reprod 80: 1168-1177, 2009.

25. Neumann CA, Cao J and Manevich Y: Peroxiredoxin 1 and its role in cell signaling. Cell Cycle 8: 4072-4078, 2009.

26. Ha B, Kim EK, Kim JH, et al: Human peroxiredoxin 1 modulates TGF- 31 -induced epithelial-mesenchymal transition through its peroxidase activity. Biochem Biophys Res Commun 421: 33-37, 2012.

27. Chen X, Halberg RB, Burch RP and Dove WF: Intestinal adenomagenesis involves core molecular signatures of the epithelial-mesenchymal transition. J Mol Histol 39: 283-294, 2008

28. Lee DJ, Kang DH, Choi M, et al: Peroxiredoxin-2 represses melanoma metastasis by increasing E-Cadherin/beta-Catenin complexes in adherens junctions. Cancer Res 73: 4744-4757, 2013 .
29. Lu W, Fu Z, Wang H, Feng J, Wei J and Guo J: Peroxiredoxin 2 is upregulated in colorectal cancer and contributes to colorectal cancer cells' survival by protecting cells from oxidative stress. Mol Cell Biochem 387: 261-270, 2014.

30. Lu W, Fu Z, Wang H, Feng J, Wei J and Guo J: Peroxiredoxin 2 knockdown by RNA interference inhibits the growth of colorectal cancer cells by downregulating Wnt/ $\beta$-catenin signaling. Cancer Lett 343: 190-199, 2014

31. Johnson RM, Ho YS, Yu DY, et al: The effects of disruption of genes for peroxiredoxin-2, glutathione peroxidase-1, and catalase on erythrocyte oxidative metabolism. Free Radic Biol Med 48: 519-525, 2010.

32. Shin SY, Kim CG, Jho EH, et al: Hydrogen peroxide negatively modulates Wnt signaling through downregulation of beta-catenin. Cancer Lett 212: 225-231, 2004.

33. Onder TT, Gupta PB, Mani SA, et al: Loss of E-cadherin promotes metastasis via multiple downstream transcriptional pathways. Cancer Res 68: 3645-3654, 2008.

34. Zhu QC, Gao RY, Wu W and Qin HL: Epithelial-mesenchymal transition and its role in the pathogenesis of colorectal cancer. Asian Pac J Cancer Prev 14: 2689-2698, 2013. 